\title{
Outsorcing quality control in the automotive industry
}

\author{
Robert Ulewicz, ${ }^{1, *}$ \\ ${ }^{1}$ Czestochowa University of Technology, Production Engineering and Safety Department, Armii \\ Krajowej 19B, PL 42-201 Czestochwa, Poland
}

\begin{abstract}
Providing high quality of products not covered by defective products is an important part of the supply chain between the manufacturer of the automotive parts and the final assembly company. The paper presents the results of research related to determining the effectiveness of quality control in a company producing plastic components for automotive industry at separate levels. Upon detection of nonconforming products at his plant, recepient according to the contract asked the supplier to launch the Controlled Shipping Level 1 procedures. As a result of the lack of significant improvement, there was run in the supplier's plant the Controlled Shipping Level 2 procedure realized on an outsourcing basis by specialized external company. Presented results of the research have made it possible to determine the effectiveness of carried out control activities. Carried out research, also confirmed by expert interviews, show that automotive companies aim to make their business more flexible so that they respond faster and more easily to internal and mainly external factors. What in the opinion of production and quality managers provides outsorcing of quality control.
\end{abstract}

\section{Introduction}

Market conditions, new development strategies, focusing on processes with the highest added value, encourages many companies not only from automotive industry to choose outsourcing solutions [1]. Outsourcing can occur anywhere in the entire supply chain as well as in manufacturing processes, after-sales and service operations [2,3]. In spite of the benefits associated with outsourcing flexibility there appear issues related to response time and costs associated with outsourcing activiestes control $[4,5]$. The question arises as to what with the costs of control if the control, such as final or interoperational control, is the subject to voluntary outsourcing or imposed by the supplier under a signed contract? In such case, it is very important to eliminate the opportunistic approach of all process participants (supply chain participants) to the outsourcing process itself $[6,7]$. From a productivity perspective, outsourcing can result in loss of information, asymmetry of information, and lack of knowledge on commissioned processes $[4,8]$, which in turn prevents the client from gaining proper insight into the quality issues that can arise both from outsourcing processes as well from automated supply chains and production processes

* Corresponding author: robert.ulewicz@wz.pcz.pl 
(technological) [9-12]. The issue of outsourcing of quality control in the automotive industry looks a bit different. In this industry we are more and more often dealing with a case of quality control on the basis of voluntary outsourcing or imposed outsourcing by the supplier. The control in this case is understood as measuring, examining or checking one or more control characteristic of the product as well as comparing the obtained results with the technical specifications $[19,20]$. Performing a control activity may involve a product or a process. In the analyzed case we are dealing with product control in the form of plastic mudguard. Control operations can be performed at a fixed location on a workstation or in a separate place. A permanent place of control at the end of the process is established when it is advisable to include quality control as a separate operation, e.g. in outsourcing control $[21,22]$. Products quality control is performed after key operations where it is absolutely necessary to meet specific parameters or there is a high risk of forming incompatibilities after operations where the product and exactly its parameters are input base for next operation or final product (assembly operations), before bottlenecks $[14,15]$. Industrial practice shows that in case of control of outsourcing, the place of carring out control usually is the end of the process.

\section{Control outsourcing}

Very high quality requirements are placed before any supplier of components. It's easy not to meet these requirements, and this generates considerable costs. A sudden increase in orders, process instability or operator error are sufficient that the manufacturing process become more complex. In situation where customer receives incompatible delivery, from which parts and components cannot be used in the further process of assembly, manufacturing facility has to take account with the risk of stopping the assembly line because of the lack of certainty as to the quality of components. In this case, more and more often companies themselves or by decision of the recipient employ external companies involved in quality control, among others: selection, repair and sorting of parts, components and finished products for assembly. Client-assembling plant in a crisis situation expects from the supplier immediate response and to solve the problem. Then the supplier has the opportunity to resorting to such outsourcing company, which will arrange for conducting selection in order to secure continuity of production to its customer.

Service of quality control of a third party is performed at the basis of the drawn up by the parties concerned so called specification of control. After completing the task entrusted the supplier and his customer receive a detailed report on the results of carried out control, and defective parts are separated and secured so that they do not enter the production line $[16,17,27]$.

In case of detecting at the client non-compliant parts the supplier is obliged to immediately implement the Controlled Shipping procedure (sometimes also called Restricted Shipping).Controlled Shipping process assumes the introduction of an additional $100 \%$ inspection of section at the end of the production process or outside the production line, in order to search for parts of $\mathrm{nOK}$, according to the accepted instruction. Controlled Shipping provides an immediate opportunity to protect the customer from receiving further non-compliant parts, and to the supplier gives time to find the root cause and to implement corrective actions [23-26]. Control in the process of Controlled Shipping can be visual or using specific control and measurement tools or gauges. Each time control of Controlled Shipping verifies $100 \%$ of parts and is confirmed by labeling parts, most commonly are used green labels. At the time of the imposition by the customer on the supplier the procedures of Controlled Shipping, the client defines the criteria for output.

Controlled Shipping process is divided into two variants: 
Controlled Shipping Level 1: employees, suppliers carry out controls in order to prevent the customer from receiving non-compliant products. Control is carried out according to the instruction accepted by the customer.

- Controlled Shipping Level 2: it is introduced when despite introduced CSL 1 control and verification of part by the supplier, the customer still finds nOK parts that means that the control of CSL 1 is conducted not in accordance with the instruction or carelessly.

- CSL 2 assumes that the control is performed out of the line by an external company. Cost of CSL 2 service is very large and most frequently entirely covered by the supplier. It happens that the client provides to a supplier a choice of several companies that are certified by him to provide CSL 2 service on his behalf. Sometimes it happens that CSL 2control is carried out in the area of the customer's warehouse.

There is a third option, which is an extension of CSL 2.

- Controlled Shipping Level 3: activities become necessary when the supplier, faced with anomalies picked up during a CSL 2, shows that they do not have the technical and organizational skills to resolve the anomalies. In addition to the checks included in aCLS 2, the third party company checks application of suitable corrective and improvement actions.

In case of introduction of the CSL 2 control, the CSL 1 control is carried out in parallel. The client defines input criteria from the process of Controlled Shipping Level 2, in a similar manner as in the case of CSL1 but with the remark that the client often additionally defines the duration of the control - e.g.: 20 days of control without defect. This means that when within 20 consecutive days of CSL 2 control an external company will not find any part of nOK this control may be stopped. In case where in 19 day of control the external company performing control finds incompatible part and noncompliance is confirmed by a customer, counter of selection days without defect resets and the control is carried out for 20 consecutive days with no defect. If the procedure CSL 2 or CSL 3 lasts for a long period of time this may be information for the customer that the supplier is not able to improve its own production process or make proper quality control in the process. In this case, the customer most frequently decides to implement the New Business Hold procedure, and in a final step there is a transfer of part production to another supplier.

\section{Case study}

For the analysis and evaluation of benefits from the use of external quality control of the product there was selected medium-sized company producing products made of thermoplastic materials, among other things from polyamide and ABS. The element under control is the wheel arch of the car (Figure 1). The customer requirement is to ensure the minimum process capability at the level of Cpk of 1.33 .

An important feature for the detail outside the geometrical parameters is uniformity of color and continuity of the surface. Detecting defect at the client launched at supplier, on command of the customer, the implementation the CSL 2 procedure. Functioning procedure CSL 1 proved to be insufficient. Indicated by the customer outsourcing company realizes control of so called third party. Practice shows that external companies in most cases, use visual control. Figure 1 shows variants of implementation of the procedure of Controlled Shipping. The situation before the implementation of the CSL procedure was as follows: injection molding machine operator after the opening of the matrix and pulling out molded piece made inspection directly on the workstation. In case of finding non-compliance of the product operator places incompatible molded piece in the separator of non-compliances. Products that meet the requirements are placed in special shipping boxes. We are dealing 
here with the case of alternative visual control and issuance of a two-stage evaluation of the compatible product "OK" and incompatible product "nOK". The controller (in this case, the operator is also the controller) can make two types of errors: defective product can pass up or reject good product, these errors are called: errors of the first type and errors of the second type. The second type of error found on production line in question does not detect incompatibilities and is called the supplier's risk, because the risk of handing over a defective product to the supplier is increasing drastically. This was the case in the analyzed case. The first type of error means that the control detects a defect that does not exist. This category is called the risk of the producer, as the producer runs the risk of rejection of good product. This generates costs, but from the point of risk of shipping of non-compliant product to receive this type of error is not so important. Table 1 shows a fragment of summary of the analysis of the effectiveness of visual control by the operator of the injection molding machine, by the plant employees on the position after the injection molding machine (CSL 1) and outsourcing control implemented by a third party (CSL 2) performed after the procedure CSL 1 . In this case we are dealing with double $100 \%$ quality control carried out by employees of the company and outside company.

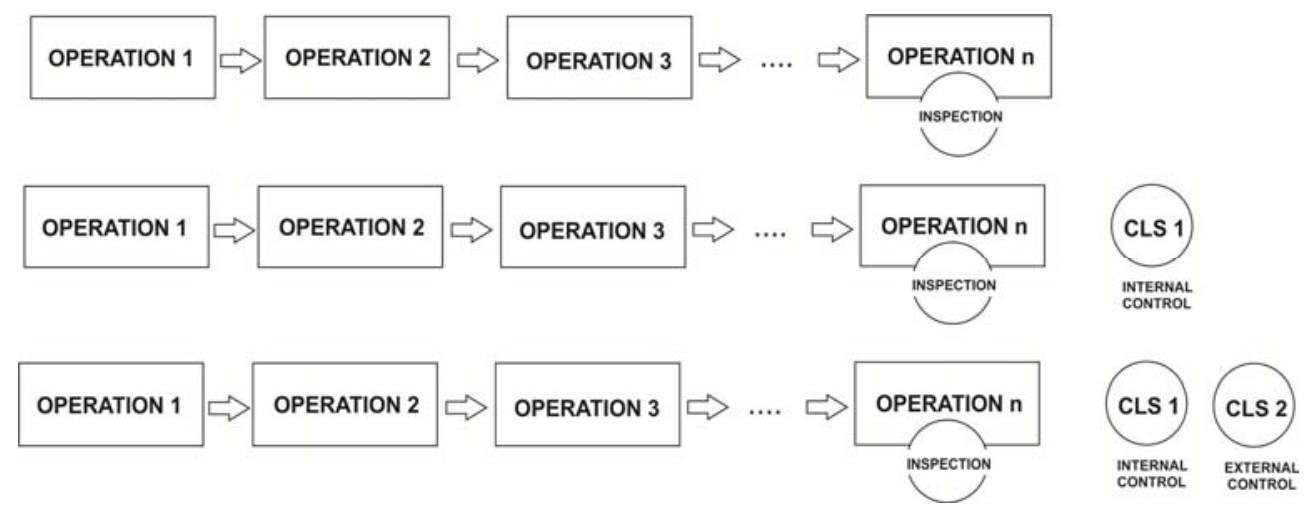

Fig. 1. Variants of implementation of the procedure of Controlled Shipping at the supplier of components made of thermoplastic materials.

Table 1. Fragment of analysis of the effectiveness of control implemented by the operator of the injection molding machine, internal controller - CSL 1 procedure and external controller - CSL 2 procedure.

\begin{tabular}{|c|c|c|c|}
\hline $\begin{array}{c}\text { Product } \\
\text { No }\end{array}$ & $\begin{array}{c}\text { Control Operator of the } \\
\text { Injection Molding } \\
\text { Machine }\end{array}$ & $\begin{array}{c}\text { Internal Controller } \\
\text { CSL1 Procedure }\end{array}$ & $\begin{array}{c}\text { External Controller } \\
\text { CSL 2 Procedure }\end{array}$ \\
\hline 1 & OK & OK & nOK \\
\hline 2 & nOK & nOK & nOK \\
\hline 3 & OK & OK & OK \\
\hline$\ldots$. & $\ldots$. & $\ldots$. & $\ldots$ \\
\hline 249 & OK & OK & nOK \\
\hline 250 & OK & OK & OK \\
\hline
\end{tabular}




\section{Conclusion}

The case analysis and the results obtained (Table 1) showed very high efficiency of used the CSL 2 control procedure, in particular in comparison with the control performed by the operator which is ineffective and worsens with the number of hours worked by the operator. At the end of the shift this type of control can cause up to $20 \%$ mistakenly identified "nOK" as "OK". At 250 carried out inspections, in case of visual control, by controllers according to CSL 1 and CSL 2 procedure, there was found $98.4 \%$ of the assessment repeatability (4 products were classified to another category). In case of comparing the results of control by operator and control realized on the basis of outsourcing (CSL 2), the repeatability of the evaluation was only $89 \%$. The benefits of using quality control on the basis of outsourcing are effective. In practice, there can be found more and more often solutions involving the resignation of quality control realized by employees of own company for outsourcing without the CSL 1.

Carried out expert interviews have revealed one more problem related to the dramatic lack of hands to work and very high turnover of employees. Especially if we are talking about voluntary rotation, which is the result of own, individual decisions of employees who have decided to change jobs. This is very difficult to control because employers do not know when they can expect such a decision and it can involve very heavy losses for the company, e.g. in the context of building a quality culture. At present, the turnover of employees in the analyzed company exceeded the level of $15 \%$ (average rotation in Poland for the first quarter of 2017 was $10.8 \%$ ).

The costs resulting from the voluntary staff rotation, including training and course costs, more increasingly convince companies from the automotive industry to use the external quality control. Using an external control company gives you a guarantee of control quality through the high competences levels of external controllers.

It is also important that companies specializing in quality control are actively involved in the process of developing suppliers and mobilizing them to improve the quality of their production, which translates explicitly into delivery of complying with the quality specification parts to the production line. The introduction of systematic quality monitoring allows protection against the consequences of human and material errors.

\section{References}

1. W.L., Tate, L.M., Ellram, L., Bals, E. Hartmann, International Journal Production Economics, 120(2), 512-524 (2009)

2. T.R. Holcomb, M.A. Hitt, Journal Operations Management, 25(2), 464-481 (2007)

3. S. Wuyts, A. Rindfleisch, A. Citrin, Outsourcing customer support: the role of provider customer focus. Journal Operations Management, 35, 40-55 (2015)

4. L.M. Ellram, W.L.Tate, C. Billington, Journal Operations Management, 26(2), 148$163(2008)$

5. J.M., Hallikas, I. Karvonen, U. Pulkkinen, VM. Virolainen, M. Tuominen, International Journal Production Econmics, 90(1), 47-58 (2004)

6. S.M. Handley, W.C. Benton Jr., Journal Operations Management, 31(3) 109-128 (2013)

7. H. Kenneth, J.B. Wathne, Journal of Marketing, 64(4), 36-51 (2000)

8. S.M. Handley, J.V. Gray, Journal Operations Management, 22(6) 1540-1556 (2013)

9. S. Borkowski, K. Knop, Production Engineering Archives, 1, 25-28 (2013)

10. H.Golas, A. Mazur, P. Piasek, P. Czajkowski. Problemy Jakości, 2, 10-14, (2017) 
11. K. Mielczarek, K. Knop, Production Engineering Archives, 13, 16-19 (2016)

12. K. Knop, K. Mielczarek, Technical Transactions Mechanics, 3, 67- 72 (2016)

13. J. Selejdak, T. Corejova, R. Ulewicz, Total Quality Management (Wz-PCz, 2016)

14. C.G. Drury, M.A. Sinclair, Human Factors, 25, 391-399 (1983)

15. A. Kujawińska, K. Vogt, Management and Production Engineering Review, 6(2), 2531 (2015)

16. A. Gębalska-Kwiecień, Proceedings of the $6^{\text {th }}$ International Conference on Operations Research and Enterprise Systems, 265-271 (2017)

17. J. Nowakowska-Grunt, M. Mazur, METAL 2016 - 25th Anniversary International Conference on Metallurgy and Materials, Conference Proceedings, 1956-1961 (2016)

18. E. Moulding: 5S: A Visual Control System for the Workplace (Author House, Central Milton Keynes 2010)

19. S.M. Handley, J.V. Gray, Journal Operations Management, 22(6), 1540-1556 (2013)

20. Y-D. Jo, K-S. Park, Journal of Loss Prevention, 16, 313-321 (2003)

21. New Trend in Global Production System

22. B.O. Örgüna, Procedia - Social and Behavioral Sciences, 181,140-147 ( 2015 )

23. J. Pietraszek, A. Gądek-Moszczak, T. Torunski, Adv. Mat. Res.-Switz., 874, 139 (2014)

24. M. Zenkiewicz, T. Zuk, J. Pietraszek, P. Rytlewski, K. Moraczewski, M. Stepczynska, Polimery, 61, 835 (2016)

25. J. Pietraszek, E. Skrzypczak-Pietraszek, Avd. Mat. Res.-Switz., 874, 151 (2014)

26. T. Zuk, J. Pietraszek, M. Zenkiewicz, Polimery, 61, 519 (2016)

27. M. Ingaldi, METAL 2017: 26 $6^{\text {th }}$ International Conference on Metallurgy and Materials, Conference Proceedings, (Brno, Czech Rep. 2017) 